\title{
Observer-Based Unknown Input Estimator of Wave Excitation Force for a Wave Energy Converter
}

\author{
Mustafa Abdelrahman and Ron Patton, Life Fellow, IEEE
}

\begin{abstract}
Several energy maximization control approaches for 2 Point Absorber Wave Energy Converter (PAWEC) systems require knowledge of the Wave Excitation Force (WEF) which is 4 not measurable during PAWEC operation. Many WEF estimators have been proposed based on stochastic PAWEC modelling using 6 the Kalman Filter (KF), Extended Kalman Filter (EKF) or receding-horizon estimation. Alternatively, a deterministic WEF 8 estimator is proposed here based on the Fast Unknown Input Estimation (FUIE) concept. The WEF is estimated as an unknown 10 input obviating the requirement to represent its dynamics. The proposed Observer-Based Unknown Input Estimator (OBUIE) 12 inherits the capability of estimating fast-changing signals from the FUIE which is important when considering irregular wave 14 conditions. Unlike preceding methods the OBUIE is designed based on a PAWEC model including the nonlinear viscous 16 drag force. It has been shown that the nonlinear viscous drag force is essential for accurate PAWEC model description, within 18 the energy maximization control role. The performance of the proposed estimator is evaluated in terms of PAWEC conversion 20 efficiency in a single degree-of-freedom PAWEC device operating in regular and irregular waves. Simulation results are obtained 22 using Matlab to evaluate the estimator under different control methods and subject to parametric uncertainty.
\end{abstract}

$24 \quad$ Index Terms-Wave Energy Conversion, Wave Excitation Force, Point Absorber, Unknown Input Observer, Monte Carlo 26 Methods

\section{INTRODUCTION}

$\mathbf{W}$ AVE energy conversion is gaining attention among marine renewable energy options thanks to high energy available from wind [1]. There is also a need for diversity are not available all the time. A disadvantage of wave power 34 is the relatively high cost of energy compared with wind power and is hence still not economically competitive [2], 36 even if the potential for technology development is high. Some investigators, e.g., [2], [3] and [4], have clarified the 38 importance of using well designed estimation and automatic control to achieve energy maximisation as a key link to 40 reduce the cost of energy. Several control techniques have been proposed to achieve wave energy conversion power maxControl (MPC) and etc., see [3]. Some methods require the sense of power maximization) control to facilitate adaptation to changing sea conditions. Some methods, e.g., MPC, even require future WEF information that can be obtained via

M. Abdelrahman and R. Patton are with the School of Engineering and Computer Science, The University of Hull, Cotingham Road, Hull, UK e-mail: (m.a.abdelrahman@2014.hull.ac.uk(M. Abdelrahman),r.j.patton@hull.ac.uk (R. Patton). prediction. However, WEF is not directly measurable during PAWEC operation and hence it should be calculated/estimated from other available or redundant PAWEC measurements.

Several studies have proposed WEF estimators based on the Kalman Filter (KF) [5], [6], [7], [8] and the Extended Kalman Filter (EKF) [9]. In general, good estimation of WEF is obtained using the KF-based approaches utilizing measurement of PAWEC output(s) and input. However, the KF-based WEF estimators necessitate the WEF dynamics to be represented in the estimator model. The WEF dynamics are usually approximated by a set of Harmonic Oscillators with a range of frequencies that should be specified based on the incoming wave, which is unknown. The number of Harmonic Oscillator frequencies and their values affect the observer accuracy as highlighted by [6][8]. In addition, the model order is noticeably increased when using KF-Based methods due to augmentation of extra states for the Harmonic Oscillator with the original system states. This adds complexity especially when considering complex PAWEC cases, e.g. multi Degree-Of-Freedom (DOF) PAWECs or PAWEC arrays. The representation of WEF dynamics using harmonic oscillators is unnecessarily complex and other approaches are proposed [10]. These authors describe a receding-horizon approach to estimate the WEF utilizing an iterative solution of a quadratic programming problem shown to give accurate estimation of WEF validated against experimental data. [10] also proposed an estimator using a KF coupled with a Random-Walk WEF model to overcome the complexity of harmonic oscillator WEF representation. The Random-Walk approach shows a comparable performance to the receding-horizon approach but with less complexity and computation burden.

As in most scientific studies two approaches to physical modelling usually exist, namely stochastic and deterministic methods. In the context of WEF estimation the former includes the KF as well as the receding horizon approaches discussed above. Here deterministic modelling methods are all based on consideration of non-linear dynamics and uncertainty. For WEF estimation this requires an understanding of the nonlinear viscous force effect (see Section II for description) which is shown to be important [11] for accurate PAWEC model utilized for energy maximization control.

Motivated by the importance of viscous force consideration for model-based energy maximization control and the fact that most of WEF estimator proposed so far have not explicitly consider this important force, this paper proposes a deterministic WEF estimator utilizing the fast unknown input estimation concept. It is worth mentioning that deterministic methods [12], [13] and the stochastic receding-horizon method [10] are based on unknown input estimation as well, but they 
all assume linear model without the viscous force term.

The contributions of this brief are (i) A novel ObserverBased Unknown Input Estimator (OBUIE) for WEF based on assumption of PAWEC model with viscous force included. The summation of the unknown excitation force and the nonlinear cous force in the motion equation of the PAWEC are considered as (a single) "unknown-input" to be estimated by the OBUIE. Then, the viscous force is calculated based on a measurement of the water surface elevation and decoupled estimation. (ii) The proposed OBUIE uses proportional and integral terms of the state estimation error in order to ensure fast and accurate estimation of the WEF considering it as an unknown input signal. (iii) Furthermore, the paper introduces analysis of the WEF estimator robustness using a Monte12 Carlo simulation, which has not been presented in previous WEF estimation studies, to the best of the authors' knowledge. This analysis spots light on the possible effect of parametric uncertainty on the PAWEC conversion efficiency when an estimator is utilized to get the WEF.

The paper is organized as follows: Section II includes a mathematical description of the PAWEC system. In Section III, the OBUIE approach for nonlinear PAWEC systems is presented. Section IV includes Matlab simulation results with nominal and perturbed PAWEC parameters. Section V (a) discusses the applicability of the OBUIE to other PAWEC types, and (b) compares the proposed OBUIE against the EKF 24 [9] and unknown input observer [12], [13] estimators. Finally, Section VI concludes the paper.

The superscript ${ }^{T}$ denotes the matrix transpose, $\|\mathbf{V}\|$ represents the Euclidean norm of a vector $\mathbf{V}$, $h e(X)=X+X^{T}$, $\star$ denotes the symmetric part of a matrix, $*$ denotes the convolution operation, and $\lambda_{\max }(X)$ and $\lambda_{\min }(X)$ refer, respectively, to the maximum and the minimum eigenvalues of a matrix $X . I_{n}$ denotes the identity matrix of dimension $n$ and $\mathbf{0}_{x \times y}$ is a matrix of dimensions $x \times y$ with zero entries.

\section{SYSTEM DESCRIPTION}

A PAWEC system consisting of a cylindrical heaving buoy of $1 / 50^{t h}$ scale and a linear generator Power Take-Off (PTO) mechanism has been constructed at the University of Hull to study wave energy conversion. See [12], [11], [14], [15] for more details about design, modeling and test of that PAWEC. The system is assumed to move only in heave. Under assumption of linear wave theory and small PAWEC motion, the dynamic oscillation is described by [16]:

$$
M \dot{v}(t)=f_{b}(t)+f_{v}(t)+f_{r}(t)+f_{e}(t)+f_{P T O}(t),
$$

where $f_{b}(t)$ is the dynamic buoyancy, $f_{v}(t)$ is the viscous force, $f_{e}(t)$ is the WEF, $f_{P T O}(t)$ is the PTO force, $\dot{v}(t)$ is the time derivative of the buoy velocity $v(t), t$ is the time in seconds and $M$ is the total mass of moving parts. Other forces such as mooring and mechanical friction are neglected.

Since a vertical cylindrical buoy is considered, $f_{b}(t)$ is proportional to the displacement $p(t)$ [15]

$$
f_{b}(t)=-K_{m} p(t),
$$

where $K_{m}$ is the hydrostatic stiffness. $K_{m}=710$ for the considered PAWEC [15]. The viscous force is given by[17]

$$
f_{v}(t)=-K_{v}\left\|v(t)-v_{w}(t)\right\|\left(v(t)-v_{w}(t)\right),
$$

where $v_{w}(t)$ is the water surface velocity near the heaving buoy, assumed measured, and $K_{v}$ is a constant that depends on the buoy geometry and buoy water interaction, for the considered PAWEC $K_{v}=50.96$ [11]. The PAWEC used here is considered a slender structure according to [17]. So that the viscous force is essential in the accurate hydrodynamic description, see [11] for more explanation.

The time domain radiation force is given by:

$$
f_{r}(t)=-M_{\infty} \dot{v}(t)-K_{r}(t) * \dot{v}(t),
$$

where $M_{\infty}$ is the added mass at infinite frequency, $M_{\infty}=$ $6.5 \mathrm{Kg}$ for the considered PAWEC [11]. $K_{r}(t)$ is the so called impulse response function, or the kernel function, of the radiation force. The term

$$
\bar{f}_{r}(t)=K_{r}(t) * v(t)=\int_{0}^{t} K_{r}(t-\tau) v(\tau) d \tau
$$

in (4) represents a convolution integral between $K_{r}(t)$ and $v(t)$ and can be approximated by a finite order state-space subsystem [18], [19] which makes the system model more suitable for control design. Hence, [14] uses the Matlab function imp2ss to directly deduce the following state-space model from the impulse response function $K_{r}(t)$ obtained using the boundary element method software NEMOH:

$$
\begin{aligned}
& \dot{x}_{r}(t)=A_{r} x_{r}(t)+B_{r} v(t), \\
& \bar{f}_{r}(t)=C_{r} x_{r}(t) .
\end{aligned}
$$

A $3^{\text {rd }}$ order model is chosen in [14] leading to:

$$
\begin{aligned}
A_{r} & =\left[\begin{array}{rrr}
-3.1848 & -4.3372 & -3.1009 \\
4.3372 & -0.0875 & -0.3882 \\
3.1009 & -0.3882 & -2.8499
\end{array}\right], \\
B_{r} & =\left[\begin{array}{lll}
-40.6964 & 5.9737 & 16.2722
\end{array}\right]^{T}, \\
C_{r} & =\left[\begin{array}{lll}
-0.4070 & -0.0597 & -0.1627
\end{array}\right] .
\end{aligned}
$$

Using (1)-(6) the PAWEC hydrodynamics can be represented in state space form as:

$$
\begin{aligned}
\dot{x}(t) & =A x(t)+B u(t)+B_{u l} f_{u l}(t), \\
y(t) & =C x(t), \\
x(t) & =\left[p(t) v(t) x_{r}(t)\right]^{T},
\end{aligned}
$$

with

$$
\begin{aligned}
& A=\left[\begin{array}{ccc}
0 & 1 & \mathbf{0}_{1 \times 3} \\
-\frac{K_{m}}{M_{t}} & 0 & -\frac{C_{r}}{M_{t}} \\
\mathbf{0}_{3 \times 1} & B_{r} & A_{r}
\end{array}\right], \\
& B=\left[\begin{array}{c}
0 \\
\frac{1}{M_{t}} \\
\mathbf{0}_{3 \times 1}
\end{array}\right], \\
& C=\left[\begin{array}{lll}
1 & 0 & \mathbf{0}_{1 \times 3} \\
0 & 1 & \mathbf{0}_{1 \times 3}
\end{array}\right], B_{u l}=B .
\end{aligned}
$$

$u(t)=f_{P T O}(t)$ is the control input, $M_{t}=M+M_{\infty}$ and

$$
f_{u l}(t)=f_{e}(t)+f_{v}(t) .
$$


$f_{u l}(t)$ represents the unknown and nonlinear part of the model.

Section III describes an observer-based technique to estimate $f_{u l}(t)$.

\section{OBSERVER-BASED UNKNOWN INPUT ESTIMATOR}

In this Section an approach for the estimation of $f_{e}(t)$ is presented, inspired by the FUIE [20], [21] strategy. This technique is used to estimate $f_{u l}(t)$ as the unknown input signal in (8).

\section{A. System Model}

Consider the model (8), where the state vector $x(t)=$ $\left[p(t) v(t) x_{r}(t)\right] \in \mathbb{R}^{5}, u(t)=f_{P T O}(t) \in \mathbb{R}^{1}$ is the control input, $f_{u l}(t) \in \mathbb{R}^{1}$ is unknown input and $y(t) \in \mathbb{R}^{2}$ is the output. The FUIE technique requires the model to satisfy the following conditions to guarantee convergent estimation [21]:

1. The state-space model is observable.

2. The rank of $C B_{u l}$ equals the number of unknown inputs, i.e., $\operatorname{rank}\left(C B_{u l}\right)=1$ in this case.

3. The invariant zeros of $\left(A, B_{u l}, C\right)$ lie on the left half s-plane.

4. The time derivative of the unknown input is normbounded, i.e., $\left\|\dot{f}_{u l}(t)\right\| \leq \bar{f} \forall t \in \mathbb{R}$ where $\bar{f}$ is non-negative.

The PAWEC model (8) with matrices (9) satisfies conditions 1-3 directly (checked using Matlab). Moreover, the force summation $f_{u l}(t)$ satisfies condition 4 , as the wave motion is continuous.
Remark 1: To facilitate the OBUIE design it is assumed that both the PAWEC position and velocity are available, and this explains the choice of matrix $C$ as in (9). This could appear as a limitation of the proposed observer. However, it is important to measure the PAWEC displacement, for example using a linear variable differential transducer (LVDT). Nevertheless, the velocity measurement is usually expensive to obtain. Here the velocity is estimated by a soft sensing using a band-pass filtered version of the low frequency position signal, since the band-pass filter is a band-limited differentiator with derivative action being most accurate at low frequencies [12]. This approach also has the advantage of filtering higher frequency noise effects. An interesting alternative is to use an inertial measurement unit which can be a cost effective way to obtain $v(t)$, see [22], for PAWEC practical implementation. However, in a real application both would be valuable to achieve suitable redundancy to enhance the PAWEC reliability in the face of inertial measurement unit malfunction.

\section{B. Observer-based WEF estimator}

The following observer is proposed for the system (8)

$$
\begin{aligned}
\dot{\hat{x}}(t) & =A \hat{x}(t)+B u(t)+B_{u l} \hat{f}_{u l}(t)-L_{1} e_{y}(t), \\
\hat{y}(t) & =C \hat{x}(t),
\end{aligned}
$$

where $\hat{x}(t) \in \mathbb{R}^{5}$ is the observer state estimate, $\hat{f}_{u l}(t)$ is the estimate of the unknown input, $\hat{y}(t) \in \mathbb{R}^{2}$ is the observer output, and $e_{y}(t):=\hat{y}(t)-y(t)$. The observer gain $L_{1}$ is designed such that $\bar{A}=A-L_{1} C$ is stable. Define the following error signals: $e_{x}(t):=\hat{x}(t)-x(t)$ and $e_{f}(t):=\hat{f}_{u l}(t)-f_{u l}(t)$, then the error dynamics are described by

$$
\dot{e}_{x}(t)=\bar{A} e_{x}(t)+B_{u l} e_{f}(t) .
$$

The following learning law, inspired from [21], is used to get $\hat{f}_{u l}(t)$ :

$$
\hat{f}_{u l}(t)=-\Gamma L_{2}\left(e_{y}(t)+\sigma \int_{0}^{t} e_{y}(\tau) d \tau\right),
$$

where $\Gamma>0$ is a learning rate, chosen by the user, and $L_{2} \quad{ }_{198}$ is a design gain.

Lemma 1 [23]: Given a scalar $\alpha>0$ and a symmetric positive definite matrix $G$, the following inequality holds:

$$
2 X^{T} Y \leq \frac{1}{\alpha} X^{T} G X+\alpha Y^{T} G^{-1} Y .
$$

Theorem 1: Under conditions 1-4 above and given positive scalars $\mu$ and $\sigma$, if there exist symmetric positive definite matrices $P \in \mathbb{R}^{5 \times 5}$ and $G \in \mathbb{R}^{1}$, such that the following constraints hold:

$$
B_{u l}^{T} P=L_{2} C
$$

and

$$
\Pi=\left[\begin{array}{cc}
\Pi_{11} & \Pi_{12} \\
\star & \Pi_{22}
\end{array}\right]<0
$$

where

$$
\begin{aligned}
& \Pi_{11}=h e(P A-Y C), \\
& \Pi_{12}=-\frac{1}{\sigma} A^{T} P B_{u l}+\frac{1}{\sigma} C^{T} Y^{T} B_{u l}, \\
& \Pi_{22}=-2 \frac{1}{\sigma} B_{u l}^{T} P B_{u l}+\frac{1}{\sigma \mu} G, \\
& Y=P L_{1},
\end{aligned}
$$

then the observer (11) and the update law (13) give the uniformly ultimately bounded estimation errors $e_{x}(t)$ and $e_{f}(t)$.

Proof: To prove the stability of the error system (12), and hence the estimation convergence, assume the following Lyapunov function

$$
V\left(\hat{x}, \hat{f}_{u l}, t\right)=e_{x}^{T}(t) P e_{x}(t)+\frac{1}{\sigma} e_{f}^{T}(t) \Gamma^{-1} e_{f}(t),
$$

then proceed to prove that $\dot{V}\left(\hat{x}, \hat{f}_{u l}, t\right)<0$. Using (12) and 200 (13) the derivative of (16) can be written as

$$
\begin{aligned}
\dot{V}\left(\hat{x}, \hat{f}_{u l}, t\right) & =e_{x}^{T}(t) h e(P \bar{A}) e_{x}(t) \\
& -e_{f}^{T}(t) h e\left(\frac{1}{\sigma} B_{u l}^{T} P B_{u l}\right) e_{f}(t) \\
& -h e\left[e_{x}^{T}(t)\left(\frac{1}{\sigma} \bar{A}^{T} P B_{u l}\right) e_{f}(t)\right] \\
& -2 e_{f}^{T}(t)\left(\frac{1}{\sigma} \Gamma^{-1}\right) \dot{f}_{u l}(t) .
\end{aligned}
$$

Using Lemma 1, it follows that

$$
\begin{aligned}
& -2 e_{f}^{T}(t)\left(\frac{1}{\sigma} \Gamma^{-1}\right) \dot{f}_{u l}(t) \\
\leq & \frac{1}{\sigma \mu} e_{f}^{T}(t) G e_{f}(t)+\frac{\mu}{\sigma} \dot{f}_{u l}^{T}(t)\left(\Gamma^{-1} G^{-1} \Gamma^{-1}\right) \dot{f}_{u l}(t), \\
\leq & \frac{1}{\sigma \mu} e_{f}^{T}(t) G e_{f}(t)+\frac{\mu}{\sigma} \dot{f}^{2} \lambda_{\max }\left(\Gamma^{-1} G^{-1} \Gamma^{-1}\right),
\end{aligned}
$$


where $\bar{f} \geq\left\|\dot{f}_{u l}(t)\right\|$ satisfies condition 4. Substituting (18) into (17), it follows that:

$$
\dot{V}\left(\hat{x}, \hat{f}_{u l}, t\right) \leq\left[\begin{array}{c}
e_{x}^{T}(t) \\
e_{f}^{T}(t)
\end{array}\right]^{T} \Pi\left[\begin{array}{l}
e_{x}(t) \\
e_{f}(t)
\end{array}\right]+\Delta
$$

where

$$
\Delta=\frac{\mu}{\sigma} \bar{f}^{2} \lambda_{\max }\left(\Gamma^{-1} G^{-1} \Gamma^{-1}\right) \geq 0,
$$

and

$$
\Pi=\left[\begin{array}{cc}
\Pi_{11} & \Pi_{12} \\
\star & \Pi_{22}
\end{array}\right]<0,
$$

with

$$
\begin{aligned}
& \Pi_{11}=h e(P A-Y C), \\
& \Pi_{12}=-\frac{1}{\sigma} A^{T} P B_{u l}+\frac{1}{\sigma} C^{T} Y^{T} B_{u l}, \\
& \Pi_{22}=-h e\left(\frac{1}{\sigma} B_{u l}^{T} P B_{u l}\right)+\frac{1}{\sigma \mu} G .
\end{aligned}
$$

Since $B_{u l}$ is full column rank, when $\Pi<0$, then (19) can be written as

$$
\dot{V}\left(\hat{x}, \hat{f}_{u l}, t\right)<-\lambda_{\min }(-\Pi)\|\zeta(t)\|^{2}+\Delta .
$$

Therefore,

$$
\dot{V}\left(\hat{x}, \hat{f}_{u l}, t\right)<0
$$

if

$$
\Delta<\lambda_{\min }(-\Pi)\|\zeta(t)\|^{2}
$$

Based on Lyapunov stability theory the estimation errors $e_{x}$ and $e_{f}$ are ultimately bounded, i.e. they converge to a small set $\Psi=\left\{\zeta(t) \mid\|\zeta(t)\|^{2} \leq \frac{\Pi}{\lambda_{\min }(-\Pi)}\right\}$ around the origin.

Actually, it is not possible to achieve asymptotic convergence, i.e. $e_{x}$ and $e_{f}$ convergence to 0 , unless the excitation force is constant or piecewise constant, which imply its first derivative is 0 valued. It is then possible to prove the asymptotic convergence using the Barbalat's Lemma. As the WEF cannot be assumed constant or even piecewise constant, it is reasonable to ensure the estimation errors converge to a small set $\Psi$. Practically $\Psi$ is close to zero and can be further tightened towards the origin by increasing the learning rate $\Gamma$ which in turn decreases $\Delta$. The small size of $\Psi$ gives confidence of the effectiveness of the estimator to get accurate enough results. This is the case as we can see from the simulation results.

Remark 2: The equality constraint (14) is proposed to simplify the proof and ensure that the optimization problem can be feasible. However, it is difficult to solve equality constraints such as (14) using the Matlab LMI toolbox. Following the idea presented in [24], (14) is replaced by

$$
\left[\begin{array}{cc}
\eta I_{5} & B_{u l}^{T} P-L_{2} C \\
\star & \eta I_{5}
\end{array}\right]>0,
$$

where $\eta$ is a positive constant chosen by the designer to be small enough to enable a good "approximation" of the equality (14) with the inequality (24). This procedure is used by a number of investigators for cases of mixed equality/inequality problems [20], [21].

After obtaining an estimate of $f_{u l}(t)$ using the OBUIE, the WEF is calculated by subtracting (3) from (10). This requires knowledge of the water surface velocity, $v_{w}(t)$, which can be obtained by differentiation of the water surface elevation measurement. The differentiation operation can be approximated using a filtered differentiation of the water surface elevation measurement, approximated by a suitable band-pass filter (see [12] and Remark 1). An alternative application approach would be to obtain $v_{w}(t)$ using an inertial measurement unit.

The solution of the LMI constraints set (24) and (15) is obtained using the Matlab LMI toolbox, with parameters $\mu=2, \eta=1 \times 10^{-4}$ and $\sigma=100$. The resulting observer gains are:

$$
L_{1}=\left[\begin{array}{cc}
0.5 & 1.0000 \\
-26.9434 & 0.1988 \\
0 & -40.5419 \\
0 & 5.9740 \\
0 & 16.3541
\end{array}\right] \text { and } L_{2}=\left[\begin{array}{ll}
0 & 22.3642
\end{array}\right] \text {. }
$$

The learning rate $\Gamma>0$ is chosen by the designer. Section IV discusses how to select a suitable value for $\Gamma$ and how the choice can influence the OBUIE performance as well as the PAWEC energy conversion efficiency.

Remark 3: The values of the scalar constants $\eta, \mu$ and $\sigma$ affect the performance of the designed observer as well as the feasibility of the LMI solution. These are chosen using trial and error, with an additional tuning requirement. However, there are some simple guidelines for design tuning: i) the variable $\eta$ should be as small as possible to ensure accurate approximation in (24). Hence, $\eta$ is set as a small positive constant less than unity. $\eta$ can then be increased gradually towards unity until the LMI feasibility is reached. ii) $\mu$ and $\sigma$ are set with small values first, for example unity, and then gradually increased to tune the design gains $L_{1}$ and $L_{2}$. It has been found that smaller values of $\mu$ and $\sigma$ cause $L_{1}$ and $L_{2}$ to have entries with small magnitudes, which means that the observer would be more stable to disturbances, e.g., measurement noise, but it may become slow and cannot capture the high frequency content of the unknown input signal. On the other hand, large values of $\mu$ and $\sigma$ result in a high-gain observer which can have fast enough dynamics to capture high frequency changes in the unknown input. However, a high-gain observer may be sensitive to disturbance effects. In summary, the designer should iterate among values of $\mu$ and $\sigma$ starting with small values seeking a balance in the designed observer between sensitivity to disturbing effects and fast response. So, the methodology here is to design (off-line) the values of the OBUIE gain matrices $L_{1}$ and $L_{2}$ to give acceptable robust performance over all considered sea states. The estimator tuning aspect is then left to an on-line speed tuning feature via the single learning rate parameter $\Gamma$.

\section{Simulation Results}

\section{A. OBUIE performance assessment and tuning}

In this part of the simulation study the OBUIE performance evaluation and the tuning of its learning rate $\Gamma$ are illustrated under irregular wave conditions. Three wave states taken from the PM spectrum are considered. The considered wave states 


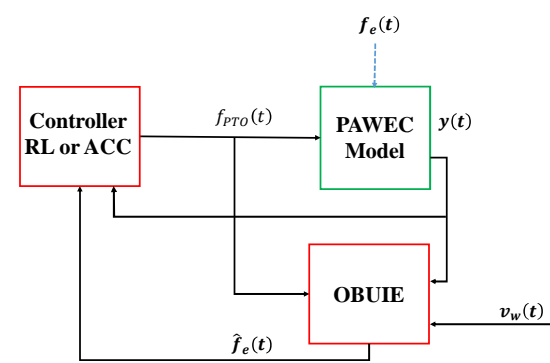

Figure 1: PAWEC control and estimation architecture

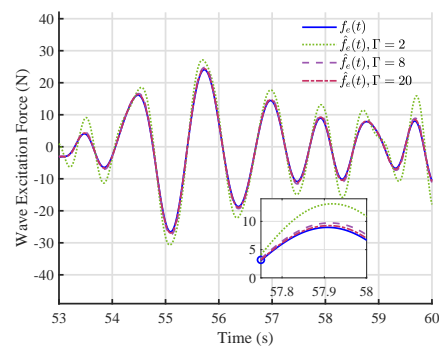

Figure 2: WEF estimations under irregular PM wave state $\left(H_{s}=0.07 \mathrm{~m}, \omega_{p}=4 \mathrm{rad} / \mathrm{s}\right)$ at $\Gamma=2,8$ and 20

$\mathrm{S} 1, \mathrm{~S} 2$ and S3 have $\omega_{p}=4 \mathrm{rad} / \mathrm{s}$ and $H_{s}=0.07 \mathrm{~m}, 0.11 \mathrm{~m}$ and $0.15 \mathrm{~m}$, respectively. The PAWEC control and estimation architecture used in the simulation study is shown in Fig. 1.

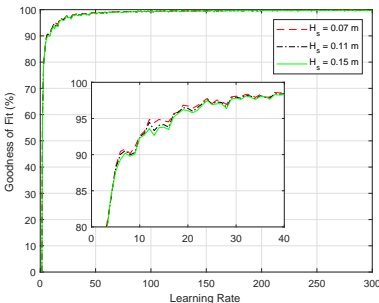

(a)

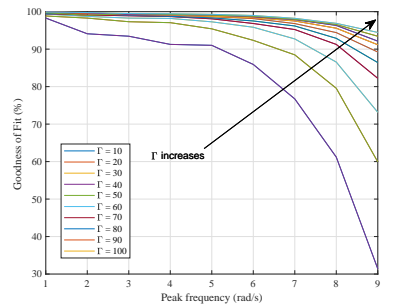

(b)
Figure 3: Illustration of the effect of changing (a) the significant wave height and (b) the peak frequency on the GoF, at different learning rates

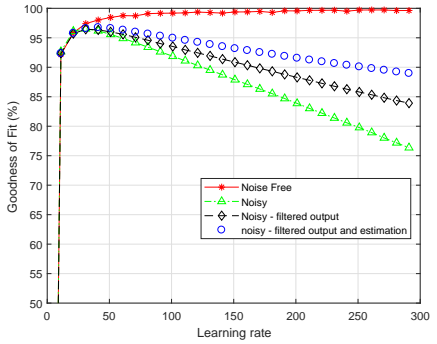

Figure 4: Measurement noise effect on the GoF with and without filtering under irregular PM wave state $\left(H_{s}=0.07 \mathrm{~m}\right.$, $\left.\omega_{p}=4 \mathrm{rad} / \mathrm{s}\right)$

(ACC) control method [25]. Figure 2 shows the comparison of the estimated and exact WEF values with learning rates: 2,8 and 20. It is assumed that the exact WEF is available in simulation, although the actual WEF cannot be available in a real experiment [12]. Fig. 2 shows that the estimated WEF accuracy improves by increasing $\Gamma$. GoF values, calculated using (25), at the three considered values of $\Gamma$ are $59.5 \%$, $93.9 \%$ and $97.9 \%$, respectively.

The GoF is investigated further by varying the learning rate among a wide range: $\Gamma \in(1,300)$, and the result is presented in Fig. 3a. The three wave sates S1,S2 and S3 are considered. At $\Gamma>100$ the $\mathrm{GoF}$ is very close to $100 \%$ for the 3 wave states. By zooming in for $\Gamma \leq 40$, it appears that for $\Gamma \geq 20$ a $\mathrm{GoF} \geq 95 \%$ is obtained. In addition, wave states with smaller significant wave heights (e.g., $0.07 \mathrm{~m}$ here) produce slightly better GoF compared with those of larger significant wave heights (e.g., $0.15 \mathrm{~m}$ here). Figure $3 \mathrm{~b}$ illustrates the relation between the GoF and the peak frequency of incoming wave at $\Gamma \in(10,100)$. Fig. 3b shows that the GoF is high at lower peak frequencies and gradually declines as the peak frequency increases. This suggests that the observer "speed" should be high enough to catch the fast-changing WEF frequencies, particularly at high peak frequency sea states. To prove this, $\Gamma$ is gradually increased from 10 to 100 . It is clear that increasing $\Gamma$ rises the GoF over all frequency range. Fore example, $\Gamma \geq 50$ is enough to make the GoF $>90$ over the whole frequency range.

To illustrate the effect of measurement noise on the estimator accuracy, white noise signals each with zero mean and variance $1 \times 10^{-7}$ are added to each of the position and velocity outputs. In Fig. 4 the GoF is plotted against $\Gamma$ for four cases: (a) noise-free outputs, (b) noisy outputs, (c) filtered noisy outputs, and filtered noisy outputs with filtered estimation. In the noisy output case, the GoF increases with $\Gamma$ till a certain point, around $\Gamma=50$, then decreases as $\Gamma$ increase. At $\Gamma=100$, for example, a $8 \%$ drop occurs in GoF due to noise. However, filtering $y(t)$, e.g. using a low-pass filter, improves the GoF. Furthermore, filtering of both $y(t)$ and $\hat{f}_{u l}$ gives rise to additional $\mathrm{GoF}$ enhancement.

The above analysis indicates that higher GoF is obtained by increasing $\Gamma$ when the output is noise-free. However, high $\Gamma$ will increase the sensitivity of the OBUIE to external disturbances, e.g., measurement noise, hence it can deteriorate the OBUIE accuracy. So that, a balance between sensitivity 


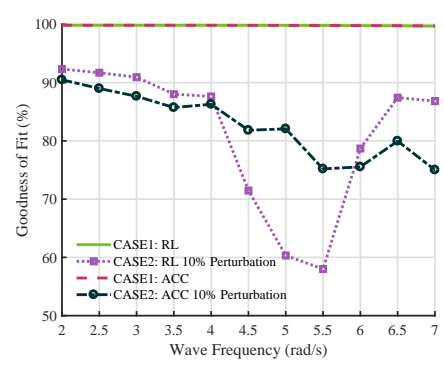

Figure 5: Average Monte-Carlo simulation for GoF at regular waves for RL and ACC and with nominal and perturbed models

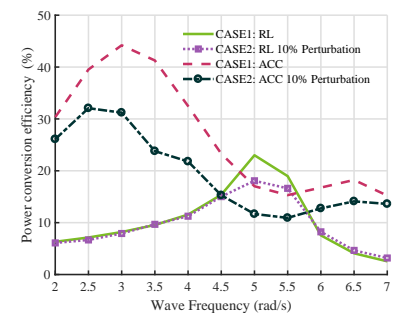

(a)

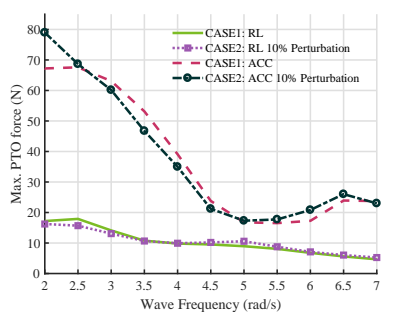

(b)
Figure 6: Average Monte-Carlo simulation for (a) conversion efficiency, and (b) maximum PTO force at regular waves for RL and ACC and with nominal and perturbed models

and accuracy should be considered while tuning $\Gamma$. In addition, suitable filtering of measurement data and estimated WEF is shown to improve the GoF.

\section{B. Effects of control strategy and parametric uncertainty}

This part of the simulation investigates the effect of using different control strategies on the performance of the proposed OBUIE as well as on the PAWEC performance in terms of: power conversion efficiency and amplitudes of the required PTO force. The conversion efficiency, also known as the relative capture width [2], is defined as:

$$
\eta_{p w r}=\bar{P}_{\text {Mech }} / P_{\text {Wave }}
$$

where $P_{W a v e}$ is the incident wave power on the buoy:

$$
P_{\text {Wave }}=2 r_{b} J .
$$

$J=\frac{\rho g^{2}}{32 \pi} T H^{2}$ is known as the capture width [16], and it represents the wave power per unit width (one meter) of incident wave, $T=2 \pi / \omega$ is the wave period, and $H$ is the wave height. The average mechanical power captured by the buoy is given by:

$$
\bar{P}_{M e c h}=\frac{1}{T_{s}} \int_{0}^{T_{s}} f_{P T O}(t) v(t) d t .
$$

where $T_{s}$ is the simulation time.

Note: A regular wave state is defined in terms of wave height:
Two control strategies are considered to provide a suitable $f_{P T O}(t)$ as a control signal, see Fig. 1. (1) The basic Resistive
Table I: A $10 \%$ parameters perturbation

\begin{tabular}{lll}
\hline Parameter & Nominal Value [15] & Perturbed Values \\
\hline$K_{m}$ & 710 & $\mathcal{N}(710,71)$ \\
$M_{t}$ & 26.5 & $\mathcal{N}(26.5,2.65)$ \\
\hline
\end{tabular}

Loading (RL) [25] strategy where the PTO force can have only a damping term and energy can only flow in the direction from the heaving buoy to the PTO. (2) The more advanced ACC method [25] which is a form of Reactive Control (RC) [16] where the PTO can have both damping and stiffness effects. Under reactive control, energy can flow in both directions between the heaving buoy and the PTO mechanism. Relative to the RL, the ACC is known to amplify both the PTO force and the system motion (i.e., displacement and velocity). This results in the amplification of the effect of the nonlinear PAWEC dynamics (e.g., the nonlinear viscous drag force). In this part of the simulation harmonic (regular) waves with frequency range: $2-7 \mathrm{rad} / \mathrm{s}$ and wave height $0.07 \mathrm{~m}$ are considered.

In addition, the effect of parametric uncertainty that may arise in the system due to modeling errors, component ageing problems or possibly extreme wave states, is investigated. Hence, some parametric uncertainties are intentionally introduced in the values of PAWEC parameters $K_{m}$ and $M_{t}$. To simulate the stochastic nature of the parametric uncertainty, the simulated parameter variations are sampled from a normal distribution with mean value equal to the nominal value and with a standard deviation of $10 \%$ of nominal value. See Table I for the stochastic values of perturbed PAWEC parameters. The Monte-Carlo experiment is used to get consistent outcomes about the stochastic uncertainty. This is achieved by running the simulation for 50 times (1000s each) with learning rate $\Gamma=20$ considering concurrently all the randomly sampled parameter variations described above. Then, mean values for: GoF, conversion efficiency, and maximum of PTO force, are computed. So, the following simulation results include two cases: CASE 1) refers to the nominal model parameters, and CASE 2) refers to model with parametric uncertainty.

The GoF is shown in Fig. 5 for both RL and ACC controllers with and without parametric uncertainty. The figure shows that the GoF is high with both RL and ACC in the nominal case (CASE 1). So that, the OBUIE almost gives similar performance with both control types. On the other hand, with the uncertain model (CASE 2) parametric perturbations cause (in average) a noticeable decline in GoF compared with the nominal case. The decline in GoF under uncertainty is more significant near resonance, i.e., around $5 \mathrm{rad} / \mathrm{s}$, with minimum values of about $58 \%$ for RL and about $75 \%$ with ACC. In the lower frequency range, $\omega \leq 4 \mathrm{rad} / \mathrm{s}$, the $\mathrm{GoF}$ is above $85 \%$ with both RL and ACC. In the higher frequency range, $\omega \geq 6 \mathrm{rad} / \mathrm{s}$, the $\mathrm{GoF}$ is $\geq 75 \%$ with $\mathrm{ACC}$ and $\geq 85 \%$ for RL. With uncertainty, the RL shows a relatively better performance than the ACC in the off-resonance regions.

Figure 6a illustrates the conversion efficiency against wave frequency. It is clear that the ACC gives higher efficiency than the RL with both nominal and perturbed models. With 


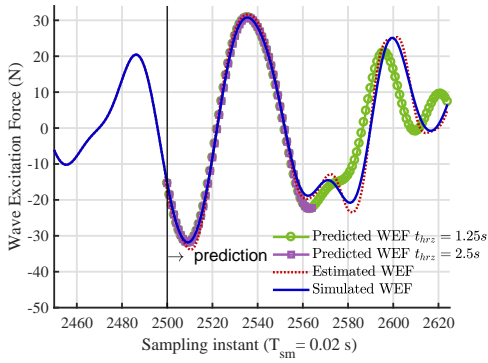

Figure 7: Prediction of WEF under irregular wave state

the nominal model, the ACC gives a maximum conversion efficiency of about $44 \%$ compared with a about $22.5 \%$ for the RL. Note that the peak efficiency points with both ACC and RL shift away from the resonance point, $5 \mathrm{rad} / \mathrm{s}$ for the considered PAWEC. This is because of considering the nonlinear viscous drag force in the PAWEC model, unlike the linear PAWEC model which always gives the peak efficiency at resonance frequency [11]. The efficiency drops due to uncertainty with both RL and ACC. Uncertainty degrades the conversion efficiency near resonance points with about $5 \%$ and $14 \%$ for RL and ACC, respectively. The decline in efficiency with the ACC is relatively high compared with the RL.

The enhanced efficiency under ACC control comes at a price of an increase in required PTO force. This is highlighted in Fig. 6b which shows that ACC requires PTO force up to $60 \mathrm{~N}$ near its peak efficiency point while it is always less than $20 \mathrm{~N}$ with RL. The higher PTO force may place restrictions on the size/rating of the PTO mechanism, an issue that should be considered early at the design stage.

\section{WEF prediction}

As highlighted in Section I, energy maximization control necessitates WEF prediction. So, WEF estimators are usually accompanied with a way to achieve WEF forecasting as described by [7], [26], [27]. It has been shown that a simple Auto-Regressive model trained with past data of WEF (or water surface elevation) can be used to accurately predict future values up to 2 significant wave periods [26]. As this technique is mature, it is combined with the OBUIE to get WEF prediction, similar to [6], [8]. An example is shown in Fig. 7 with an irregular wave sampled from the PM spectrum with: $H_{s}=0.15 \mathrm{~m}$ and $\omega_{p}=5 \mathrm{rad} / \mathrm{s}$. The Simulink sampling time is set to $0.02 \mathrm{~s}$ and the learning rate is taken as $\Gamma=7$. Two prediction horizons are considered, $t_{h r z 1}=1.25 \mathrm{~s}$ and $t_{h r z 2}=2.5 \mathrm{~s}$. It is evident from Fig. 7 that an Auto-Regressive model of order 50 can accurately predict the WEF up to one SWP in the future, with a GoF $\approx 94 \%$. However, doubling the prediction horizon, i.e., $t_{h r z 2}=2.5 \mathrm{~s}$, degrades the prediction accuracy to $\mathrm{GoF} \approx 71 \%$.

\section{Discussion}

\section{A. Applicability to other PAWEC geometries}

The proposed WEF estimator handles the (nonlinear) effect of the nonlinear viscous drag force as the considered PAWEC has a cylindrical geometry for which the nonlinear viscous drag force is significant. Other nonlinear effects arise with different geometries. For example, the (nonlinear) FroudeKrylov (FK) force is important in PAWECs with non-uniform cross section areas [28], [29], e.g., cone or sphere. Significant work is carried out by the studies [28], [29], [30] in order to provide a fast but accurate enough representation of FK force in a wide range of realistic PAWECs that can be used in Model-Based control systems design. Based on the authors' current knowledge, such models with FK force cannot yet be used in model-based control design.

In keeping with several control and estimation methods, the OBUIE is a model-based technique that depends on a certain model structure as in (8). Further investigation of (8) reveals that this structure has two parts: i) a linear part: $A x(t)+B u(t)$ and ii) (unknown + nonlinear) part: $B_{u l} f_{u l}$. The second part includes $f_{e}(t)$ plus nonlinear term(s), e.g., a nonlinear viscous drag force. For the OBUIE applicability to a certain PAWEC with any degrees of freedom or geometries, It is required (a) to represent the PAWEC dynamics model in the form of (8) (b) to have suitable information to calculate the nonlinear term(s), i.e., decouple the nonlinear term(s) from the (unknown + nonlinear) part. Then the OBUIE can be used to estimate the (unknown + nonlinear) part as a single accumulated unknown input. Consequently, $f_{e}(t)$ is obtained via subtraction. In this study the OBUIE is used to estimate $f_{u l}$, i.e., the (unknown + nonlinear) part, then the knowledge of $v(t)$ and $v_{w}(t)$ is used to calculate the nonlinear viscous drag force, i.e., the nonlinear term. Finally, (3) is subtracted from (10) to compute the WEF.

\section{B. Comparison with EKF and unknown input observer esti- mators}

The proposed OBUIE is shown to handle accurate nonlinear forces, e.g. viscous or friction, in the PAWEC under the availability of suitable information to decouple them from the estimation of (accumulated) unknown input. To the best of the authors' knowledge, available WEF estimators have not explicitly discussed the case of nonlinearity in the PAWEC model and in most cases reported linear models are assumed. Except in [9] where an EKF-based WEF estimator was designed for a nonlinear model. But it requires the computation of Jacobian matrices step by step to linearise the model, adding considerable computational burden. In addition, the EKF may fail if the linearized model is far from the actual nonlinear process. This is actually a well-known potential limitation of the EKF which requires the determination of a domain of convergence that is difficult to determine[31], especially for a system as complex as wave motion with varying properties. This is in agreement with [6] where the authors reported that EKF is limited to regular wave and it may diverge when applied to irregular wave scenarios. However, it is possible that some available WEF estimators such as [10] and [12] could be extended to handle nonlinear terms in a similar fashion as presented in this paper.

The OBUIE has some similarity with the unknown input observer based estimators in [12] and [13], as both do not need representation of WEF dynamics in the estimation model. 
However, the OBUIE has more flexibility than [12] and [13] as

the user can tune a learning rate $(\Gamma)$ to increase the speed of the observer and hence capture possibly fast-changing WEF dynamics, leading to better estimation accuracy. In contrast, the speed of the WEF estimators proposed in [12], [13] is determined during the (off-line) design stage using an LMIbased strategy for pole-assignment. The downside is that the LMI design may fail to find a feasible solution.

\section{CONCLUSION AND FUTURE WORK}

The paper presents the OBUIE technique to estimate the WEF on a single DOF PAWEC. Unlike some WEF estimators available in the literature, the proposed OBUIE has a simple structure as it does not need a representation of the WEF dynamics in the estimator. It is also able to handle the nonlinear effect due to viscous force but requires the knowledge of the water surface velocity. Finally, the OBUIE does not require heavy computation. The Matlab simulation results show the OBUIE validity to estimate the WEF corresponding to basic (RL) and advanced (ACC) controllers, and subject to both regular and irregular wave conditions. Under parametric perturbations, the OBUIE continues to deliver acceptable WEF estimation but with reduced PAWEC conversion efficiency. This comes as a consequence of parametric uncertainty. The OBUIE is based on the cylindrical-shaped PAWEC dynamics. Further analysis is ongoing to study the applicability of OBUIE on PAWECs with complex geometries and multi-DOF, where more nonlinear effects may arise and be signification.

\section{ACKNOWLEDGEMENT}

Mustafa Abdelrahman thanks the British Council and the Egyptian Government for joint PhD scholarship.

\section{REFERENCES}

[1] A. Clément, P. McCullen, A. Falcão, A. Fiorentino, F. Gardner, K. Hammarlund, G. Lemonis, T. Lewis, K. Nielsen, S. Petroncini et al., "Wave energy in europe: current status and perspectives," Renewable and sustainable energy reviews, vol. 6, no. 5, pp. 405-431, 2002.

[2] F. Fusco and J. V. Ringwood, "A simple and effective real-time controller for wave energy converters," IEEE Trans. Sustain. Energy, vol. 4, no. 1, pp. 21-30, 2013.

[3] J. V. Ringwood, G. Bacelli, and F. Fusco, "Energy-maximizing control of wave-energy converters: The development of control system technology to optimize their operation," IEEE Control Systems, vol. 34, no. 5, pp. 30-55, 2014.

[4] U. A. Korde and J. V. Ringwood, Hydrodynamic Control of Wave Energy Devices. Cambridge University Press, 2016.

[5] P. Kracht, S. Perez-Becker, J.-B. Richard, and B. Fischer, "Performance improvement of a point absorber wave energy converter by application of an observer-based control: Results from wave tank testing," IEEE Transactions on Industry Applications, vol. 51, no. 4, pp. 3426-3434, 2015.

[6] M. Garcia-Abril, F. Paparella, and J. Ringwood, "Excitation force estimation and forecasting for wave energy applications," IFACPapersOnLine, vol. 50, no. 1, pp. 14 692-14 697, 2017.

[7] B. A. Ling and B. A. Batten, "Real time estimation and prediction of wave excitation forces on a heaving body," in ASME 2015 34th international conference on ocean, offshore and arctic engineering. American Society of Mechanical Engineers, 2015, pp. V009T09A017V009T09A017.

[8] Y. Peña-Sanchez, M. Garcia-Abril, F. Paparella, and J. V. Ringwood, "Estimation and forecasting of excitation force for arrays of wave energy devices," IEEE Transactions on Sustainable Energy, vol. 9, no. 4, pp. $1672-1680$, Oct 2018.
[9] O. Abdelkhalik, S. Zou, R. Robinett, G. Bacelli, and D. Wilson, "Estimation of excitation forces for wave energy converters control using pressure measurements," International Journal of Control, vol. 90, no. 8, pp. 1793-1805, 2017.

[10] H. N. Nguyen and P. Tona, "Wave excitation force estimation for wave energy converters of the point-absorber type," IEEE Transactions on Control Systems Technology, pp. 1-9, 2018.

[11] S. Jin, R. J. Patton, and B. Guo, "Viscosity effect on a point absorber wave energy converter hydrodynamics validated by simulation and experiment," Renewable Energy, vol. 129, pp. 500 - 512, 2018.

[12] B. Guo, R. J. Patton, S. Jin, and J. Lan, "Numerical and experimental studies of excitation force approximation for wave energy conversion," Renewable Energy, vol. 125, pp. 877-889, 2018.

[13] M. Abdelrahman and R. Patton, "Robust control of a wave energy converter with soft sensing of wave excitation force," in 12th European Wave and Tidal Energy Conference (EWTEC 2017), Cork, Ireland, 2017.

[14] B. Guo, R. Patton, S. Jin, J. Gilbert, and D. Parsons, "Nonlinear modeling and verification of a heaving point absorber for wave energy conversion," IEEE Transactions on Sustainable Energy, vol. 9, no. 1, pp. 453-461, 2018.

[15] B. Guo, R. Patton, M. Abdelrahman, and J. Lan, "A continuous control approach to point absorber wave energy conversion," in 2016 UKACC 11th International Conference on Control, Aug 2016, pp. 1-6.

[16] J. Falnes, Ocean waves and oscillating systems: linear interactions including wave-energy extraction. Cambridge university press, 2002.

[17] M. E. McCormick, Ocean wave energy conversion. Courier Corporation, 2013.

[18] Z. Yu and J. Falnes, "State-space modelling of a vertical cylinder in heave," Applied Ocean Research, vol. 17, no. 5, pp. 265-275, 1995.

[19] R. Taghipour, T. Perez, and T. Moan, "Hybrid frequency-time domain models for dynamic response analysis of marine structures," Ocean Engineering, vol. 35, no. 7, pp. 685-705, 2008

[20] K. Zhang, B. Jiang, and V. Cocquempot, "Fast adaptive fault estimation and accommodation for nonlinear time-varying delay systems," Asian Journal of Control, vol. 11, no. 6, pp. 643-652, 2009.

[21] — "Adaptive observer-based fast fault estimation," International Journal of Control, Automation, and Systems, vol. 6, no. 3, pp. 320326, 2008

[22] N. O. Abankwa, S. J. Johnston, M. Scott, and S. J. Cox, "Ship motion measurement using an inertial measurement unit," in 2015 IEEE 2nd World Forum on Internet of Things (WF-IoT), Dec 2015, pp. 375-380.

[23] B. Jiang, J. L. Wang, and Y. C. Soh, "An adaptive technique for robust diagnosis of faults with independent effects on system outputs," International Journal of Control, vol. 75, no. 11, pp. 792-802, 2002.

[24] M. Corless and J. Tu, "State and input estimation for a class of uncertain systems," Automatica, vol. 34, no. 6, pp. 757-764, 1998.

[25] J. Hals, J. Falnes, and T. Moan, "A comparison of selected strategies for adaptive control of wave energy converters," Journal of Offshore Mechanics and Arctic Engineering, vol. 133, no. 3, p. 031101, 2011.

[26] F. Fusco and J. V. Ringwood, "Short-term wave forecasting for real-time control of wave energy converters," IEEE Transactions on sustainable energy, vol. 1, no. 2, pp. 99-106, 2010.

[27] M. P. Schoen, J. Hals, and T. Moan, "Wave prediction and robust contro of heaving wave energy devices for irregular waves," IEEE Transactions on Energy Conversion, vol. 26, no. 2, pp. 627-638, 2011.

[28] G. Giorgi and J. V. Ringwood, "Nonlinear froude-krylov and viscous drag representations for wave energy converters in the computation/fidelity continuum," Ocean Engineering, vol. 141, pp. 164-175, 2017.

[29] — "Computationally efficient nonlinear froude-krylov force calculations for heaving axisymmetric wave energy point absorbers," Journal of Ocean Engineering and Marine Energy, vol. 3, no. 1, pp. 21-33, 2017.

[30] _ - "Analytical representation of nonlinear froude-krylov forces for 3-dof point absorbing wave energy devices," Ocean Engineering, vol. 164, pp. 749-759, 2018.

[31] M. Boutayeb, H. Rafaralahy, and M. Darouach, "Convergence analysis of the extended kalman filter used as an observer for nonlinear deterministic discrete-time systems," IEEE transactions on automatic control, vol. 42, no. 4, pp. 581-586, 1997. 